\title{
13 Integral Equations
}

\subsection{Introduction}

The Green's function method can transform a differential equation into an integral equation if the source term actually contains the solution being sought. For example, in the quantum theory of scattering the Schrödinger equation can be cast in the form

$$
\left(\nabla^{2}+k^{2}\right) \psi(\boldsymbol{r})=U(\boldsymbol{r}) \psi(\boldsymbol{r})
$$

where $U(\boldsymbol{r})=\frac{2 m}{\hbar^{2}} V(\boldsymbol{r})$ and $V(\boldsymbol{r})$ is the potential describing the interaction responsible for the scattering. If we describe the incident particle by means of a plane wave $e^{i \boldsymbol{k}_{0} \cdot \boldsymbol{r}}$ where $\left|\boldsymbol{k}_{0}\right|=k$, the solution of (13.1.1) that we seek must satisfy the boundary condition

$$
\psi(\boldsymbol{r}) \rightarrow e^{i \boldsymbol{k}_{0} \cdot \boldsymbol{r}}+f(\theta, \varphi) \frac{e^{i k r}}{r} \text { as } r \rightarrow \infty
$$

As we learned in an earlier discussion of scattering, the second term of this expression is the outgoing spherical scattered wave produced by the interaction. The Green's function appropriate to such a problem is the outgoing Green's function for the Helmhotz equation given by (12.3.15):

$$
G\left(\boldsymbol{r} ; \boldsymbol{r}^{\prime}\right)=-\frac{1}{4 \pi} \frac{e^{i k\left|\boldsymbol{r}-\boldsymbol{r}^{\prime}\right|}}{\left|\boldsymbol{r}-\boldsymbol{r}^{\prime}\right|}
$$

Using it with a source function $\sigma(\boldsymbol{r})=U(\boldsymbol{r}) \psi(\boldsymbol{r})$ and imposing the boundary condition (13.1.2), we see that the desired solution of the PDE can be expressed as

$$
\psi(\boldsymbol{r})=e^{i \boldsymbol{k}_{0} \cdot \boldsymbol{r}}-\int_{\text {all }} \frac{e^{i k\left|\boldsymbol{r}-\boldsymbol{r}^{\prime}\right|}}{\left|\boldsymbol{r}-\boldsymbol{r}^{\prime}\right|} U\left(\boldsymbol{r}^{\prime}\right) \psi\left(\boldsymbol{r}^{\prime}\right) d V^{\prime} .
$$

Notice that this integral equation incorporates both the PDE and the boundary condition that we wish to impose. This is a standard feature of integral equations and one that often renders them a more convenient or more powerful tool than differential equations. Add to this the fact that some problems, notably those involving transport phenomena, admit solution only by means of integral equations and we conclude that they warrant some attention. Since any multi-dimensional integral equation can be reduced to an equivalent set of one-dimensional equations by making use of eigenfunction expansions, we shall focus that attention on the one-dimensional case.

\subsection{Types of Integral Equations}

Integral equations are classified in terms of their integration limits and in terms of whether the unknown function appears inside and outside the integral or only outside. We call equations with fixed integration limits Fredholm equations. Those with 
one limit that is variable are called Volterra equations. If the unknown function appears only under the integral sign, the equation is said to be an integral equation of the first kind; otherwise it is an equation of the second kind.

Thus,

1.

$$
f(x)=\int_{a}^{b} K(x, y) \psi(y) d y
$$

is a Fredholm equation of the first kind;

2.

$$
\psi(x)=f(x)+\lambda \int_{a}^{b} K(x, y) \psi(y) d y, \quad \lambda=\text { a constant }
$$

is a Fredholm equation of the second kind;

3.

$$
f(x)=\int_{a}^{x} K(x, y) \psi(y) d y
$$

is a Volterra equation of the first kind; and

4.

$$
\psi(x)=f(x)+\int_{a}^{x} K(x, y) \psi(y) d y
$$

is a Volterra equation of the second kind.

We say that (13.2.2) and (13.2.4) are homogeneous if $f(x) \equiv 0$. In all four cases $f(x)$ and $K(x, y)$ are presumed to be known and $\psi(y)$ is the function whose identity is sought. As with integral representations, $K(x, y)$ is called the kernel.

Evidently, equation (13.1.3) is a (three-dimensional) Fredholm equation of the second kind. Volterra equations arise when the solution and its first derivative are specified at a single point as is the case with initial conditions. For example, if we start with the second-order non-homogeneous DE

$$
\frac{d^{2} \psi}{d x^{2}}+a(x) \frac{d \psi}{d x}+b(x) \psi(x)=g(x)
$$

complimented by (initial) conditions $\psi\left(x_{0}\right)=u_{0}$ and $\psi^{\prime}\left(x_{0}\right)=v_{0}$, we can use indefinite integration to write

$$
\frac{d \psi}{d x}=-\int_{x_{0}}^{x} a(x) \frac{d \psi}{d x} d x-\int_{x_{0}}^{x} b(x) \psi(x) d x+\int_{x_{0}}^{x} g(x) d x+v_{0}
$$


Integrating the first integral on the right by parts gives us

$$
\frac{d \psi}{d x}=-a(x) \psi(x)-\int_{x_{0}}^{x}\left[b(x)-a^{\prime}(x)\right] \psi(x) d x+\int_{x_{0}}^{x} g(x) d x+a\left(x_{0}\right) u_{0}+v_{0}
$$

which incorporates both of our initial conditions. Integrating the complete equation a second time, we obtain

$$
\begin{aligned}
\psi(x)= & -\int_{x_{0}}^{x} a(x) \psi(x) d x-\int_{x_{0}}^{x} \int_{x_{0}}^{x}\left[b(y)-a^{\prime}(y)\right] \psi(y) d y d x \\
& +\int_{x_{0}}^{x} \int_{x_{0}}^{x} g(y) d y d x+\left[a\left(x_{0}\right) u_{0}+v_{0}\right]\left(x-x_{0}\right)+u_{0} .
\end{aligned}
$$

This can be simplified by using the identity ( the two sides have the same first derivative and the same value at $x_{0}$ )

$$
\int_{x_{0}}^{x} \int_{x_{0}}^{x} f(y) d y d x=\int_{x_{0}}^{x}(x-y) f(y) d y
$$

Applying it to (13.2.6) we find

$$
\begin{aligned}
\psi(x)= & -\int_{x_{0}}^{x}\left\{a(y)+(x-y)\left[b(y)-a^{\prime}(y)\right]\right\} \psi(y) d y \\
& +\int_{x_{0}}^{x}(x-y) g(y) d y+\left[a\left(x_{0}\right) u_{0}+v_{0}\right]\left(x-x_{0}\right)+v_{0} .
\end{aligned}
$$

Evidently, this is a Volterra integral equation of the second kind and comparing it with (13.2.4) we can identify

$$
K(x, y) \equiv(y-x)\left[b(y)-a^{\prime}(y)\right]-a(y)
$$

as the kernel and

$$
f(x) \equiv \int_{x_{0}}^{x}(x-y) g(y) d y+\left[a\left(x_{0}\right) u_{0}+v_{0}\right]\left(x-x_{0}\right)+u_{0}
$$

as the non-homogeneous term.

\subsection{Convolution Integral Equations}

If the kernel and integration limits of a Fredholm equation are those of a known integral transform then it can be solved simply by invoking the corresponding inverse 
transform. Beyond this almost trivial application, an integral transform can also be useful in the solution of an integral equation when the kernel has the symmetry properties needed to exploit the transform's convolution theorem. For example, suppose that we have a Fredholm equation of the form

$$
\psi(x)=f(x)+\int_{-\infty}^{\infty} K(x-y) \psi(y) d y
$$

where the kernel depends only on the difference between the variables $x$ and $y$. The integral is a convolution integral of the type that arises for Fourier transforms. Therefore, when we transform the equation we find

$$
\Psi(k)=F(k)+2 \pi \kappa(k) \Psi(k)
$$

where

$$
\begin{aligned}
\Psi(k) & \equiv \mathcal{F}\{\psi(x)\}=\frac{1}{\sqrt{2 \pi}} \int_{-\infty}^{\infty} f(x) e^{i k x} d x, \\
F(k) & \equiv \mathcal{F}\{f(x)\}=\frac{1}{\sqrt{2 \pi}} \int_{-\infty}^{\infty} f(x) e^{i k x} d x
\end{aligned}
$$

and

$$
\kappa(k) \equiv \mathcal{F}\{K(x)\}=\frac{1}{\sqrt{2 \pi}} \int_{-\infty}^{\infty} K(x) e^{i k x} d x .
$$

Solving (13.3.2), we have

$$
\Psi(k)=\frac{F(k)}{1-2 \pi \kappa(k)}
$$

and so,

$$
\psi(x)=\frac{1}{\sqrt{2 \pi}} \int_{-\infty}^{\infty} \frac{F(k)}{1-2 \pi \kappa(k)} e^{-i k x} d k
$$

Laplace transforms are equally useful in the solution of Volterra equations of the form

$$
\psi(x)=f(x)+\int_{0}^{x} K(x-y) \psi(y) d y .
$$

For example, Abel's integral equation is

$$
f(x)=\int_{0}^{x} \frac{\psi(y)}{(x-y)^{\alpha}} d y, \quad 0<\alpha<1
$$


where $f(x)$ is known but $\psi(x)$ is not. The left hand side of this equation is a convolution integral of the type that arises with Laplace transforms and taking the transform of both sides of the equation, we obtain

$$
\mathcal{L}\{f(x)\}=\mathcal{L}\left\{x^{-\alpha}\right\} \mathcal{L}\{\psi(x)\} .
$$

Since $\mathcal{L}\left\{x^{-\alpha}\right\}=\frac{\Gamma(1-\alpha)}{s^{1-\alpha}}$, this means that

$$
\mathcal{L}\{\psi(x)\}=\frac{s^{1-\alpha} \mathcal{L}\{f(x)\}}{\Gamma(1-\alpha)} .
$$

The inverse $\mathcal{L}^{-1}\left\{s^{1-\alpha}\right\}$ does not exist but $\mathcal{L}^{-1}\left\{s^{-\alpha}\right\}=\frac{\chi^{\alpha-1}}{\Gamma(\alpha)}$. Therefore, we divide through by $s$ to obtain

$$
\frac{1}{s} \mathcal{L}\{\psi(x)\}=\frac{\mathcal{L}\left\{x^{\alpha-1}\right\} \mathcal{L}\{f(x)\}}{\Gamma(\alpha) \Gamma(1-\alpha)}=\frac{\sin \pi \alpha}{\pi} \mathcal{L}\left\{x^{\alpha-1}\right\} \mathcal{L}\{f(x)\}
$$

where we have invoked the gamma function identity (5.2.10). This can be inverted by means of a further application of the convolution theorem which yields

$$
\int_{0}^{x} \psi(y) d y=\frac{\sin \pi \alpha}{\pi} \int_{0}^{x} \frac{f(y)}{(x-y)^{1-\alpha}} d y,
$$

or

$$
\psi(x)=\frac{\sin \pi \alpha}{\pi} \frac{d}{d x} \int_{0}^{x} \frac{f(y)}{(x-y)^{1-\alpha}} d y
$$

\subsection{Integral Equations With Separable Kernels}

Fredholm equations of the second kind can be solved in closed form if their kernels are separable, that is if they have the form

$$
K(x, y)=u(x) v(y) .
$$

To demonstrate this we substitute (13.4.1) into (13.2.2) to obtain

$$
\psi(x)=f(x)+\lambda \int_{a}^{b} K(x, y) \psi(y) d y=f(x)+\lambda \int_{a}^{b} u(x) v(y) \psi(y) d y=f(x)+\lambda C u(x)
$$

where

$$
C=\int_{a}^{b} v(y) \psi(y) d y
$$


Next, we substitute the final line of (13.4.2) into (13.4.3) to create a linear equation for $C$ :

$$
C=\int_{a}^{b} v(y)[f(y)+\lambda C u(y)] d y .
$$

Solving for $C$, we find

$$
C=\frac{\int_{a}^{b} v(y) f(y) d y}{1-\lambda \int_{a}^{b} u(y) v(y) d y}
$$

which, when substituted into (13.4.2), yields the solution

$$
\psi(x)=f(x)+\lambda u(x)\left[\frac{\int_{a}^{b} v(y) f(y) d y}{1-\lambda \int_{a}^{b} u(y) v(y) d y}\right] .
$$

The homogeneous version of (13.2.2) is the eigenvalue equation

$$
\psi(x)=\lambda \int_{a}^{b} K(x, y) \psi(y) d y .
$$

This too is readily solved when the kernel is separable. Substituting (13.4.1) into it gives us

$$
\psi(x)=\lambda u(x) \int_{a}^{b} v(y) \psi(y) d y=\lambda C u(x)=(\text { constant }) \times u(x) .
$$

In this case, substitution of (13.4.7) into the definition of $C$ in (13.4.3) does not determine $C$ (because it cancels out of the resulting equation) but it does determine the eigenvalue $\lambda$ :

$$
\lambda=\left[\int_{a}^{b} u(y) v(y) d y\right]^{-1}
$$

Thus, the eigenvalue is uniquely determined and the corresponding eigenfunction is determined to within a multiplicative constant that can be fixed through a normalization condition placed on $\psi(x)$ over the interval $a \leq x \leq b$.

Notice that if $\lambda$ in (13.4.5) is equal to the eigenvalue defined by (13.4.8), $C$ is infinite and the non-homogeneous equation has no solution unless

$$
\int_{a}^{b} v(y) f(y) d y=0
$$


in which case the solution is

$$
\psi(x)=f(x)+(\text { constant }) \times u(x) .
$$

We have seen the same phenomenon arise with the solution of non-homogeneous differential equations.

Kernels that are sums of separable pieces, such as

$$
K(x, y)=\sum_{j=1}^{N} u_{j}(x) u_{j}(y),
$$

also give rise to integral equations that can be solved explicitly. To confirm this, we substitute (13.4.9) into (13.2.2) to obtain

$$
\begin{aligned}
\psi(x) & =f(x)+\lambda \int_{a}^{b} K(x, y) \psi(y) d y=f(x)+\lambda \sum_{j=1}^{N} u_{j}(x) \int_{a}^{b} v_{j}(y) \psi(y) d y \\
& =f(x)+\lambda \sum_{j=1}^{N} c_{j} u_{j}(x)
\end{aligned}
$$

where the constants $c_{j}$ are given by

$$
c_{j}=\int_{a}^{b} v_{j}(y) \psi(y) d y .
$$

Substitution of the third line of (13.4.10) in place of $\psi(y)$ under the integral in (13.4.11) generates a set of linear algebraic equations to determine the $c_{j}$ :

$$
c_{i}=\int_{a}^{b} v_{i}(y) f(y) d y+\lambda \sum_{j=1}^{N}\left[\int_{a}^{b} v_{i}(y) u_{j}(y) d y\right] c_{j} .
$$

This can be re-written in matrix notation by introducing the $n \times n$ matrix $M$ with elements

$$
M_{i j}=\int_{a}^{b} v_{i}(y) u_{j}(y) d y
$$

and the vectors $C$ and $F$ with elements $C_{i}$ and

$$
F_{i}=\int_{a}^{b} v_{i}(y) f(y) d y,
$$

respectively. Specifically, (13.4.12) becomes

$$
\sum_{j=1}^{N} \delta_{i j} c_{j}=F_{i}+\lambda \sum_{j=1}^{N} M_{i j} c_{j}
$$


or

$$
\sum_{j=1}^{N}\left(\delta_{i j}-\lambda M_{i j}\right) c_{j}=F_{i}
$$

or

$$
(\mathbb{I}-\lambda M) C=F .
$$

Thus, the values of the constants $c_{i}$ are given by

$$
C=(\mathbb{I}-\lambda M)^{-1} F
$$

and these, in turn, completely determine the solution of the non-homogeneous integral equation via equation (13.4.10). In the case of the (homogeneous) eigenvalue problem (13.4.6), equation (13.4.10) still obtains but with $f(x) \equiv 0$. Therefore, we set $F \equiv 0$ in (13.4.13) and so obtain the matrix eigenvalue problem

$$
(\mathbb{I}-\lambda M) C=0 .
$$

This has a non-trivial solution if and only if

$$
\operatorname{det}(\mathbb{I}-\lambda M)=0
$$

which determines at least one and at most $N$ eigenvalues $\lambda_{n}$. Substituting these back into (13.4.15), one can find the corresponding eigenvectors $C^{(n)}$ whose components $c_{i}^{(n)}$ determine the eigenfunction solutions of the integral equation via

$$
\psi_{n}(x)=(\text { constant }) \times \sum_{j=1}^{N} c_{j}^{(n)} u_{j}(x)
$$

By way of illustration, consider the simple example presented by the equation

$$
\psi(x)=\lambda \int_{-1}^{1}(y+x) \psi(y) d y
$$

from which we can read off $u_{1}(x)=1, v_{1}(y)=y, u_{2}(x)=x, v_{2}(y)=1$. Thus,

$$
M_{11}=M_{22}=\int_{-1}^{1} y d y=0, \quad M_{12}=\int_{-1}^{1} y^{2} d y=\frac{2}{3}, \quad M_{21}=\int_{-1}^{1} d y=2
$$

and equation (13.4.16) reads

$$
\left|\begin{array}{cc}
1 & -\frac{2 \lambda}{3} \\
-2 \lambda & 1
\end{array}\right|=0
$$


Expanding, we obtain

$$
1-\frac{4 \lambda^{2}}{3}=0
$$

and hence, $\lambda= \pm \frac{\sqrt{3}}{2}$. As we shall see in Section 13.6, the reality of the eigenvalues is a consequence of the symmetry of the kernel under $x \leftrightarrow y$. If the kernel were to be replaced by the skew-symmetric function $K(x, y)=(y-x)$, the eigenvalues would be the pure imaginary $\lambda= \pm i \frac{\sqrt{3}}{2}$. Substitution of $\lambda= \pm \frac{\sqrt{3}}{2}$ into (13.4.15) gives us

$$
c_{2}^{(1)}=\sqrt{3} c_{1}^{(1)} \text { and } c_{2}^{(2)}=-\sqrt{3} c_{1}^{(2)} \text {. }
$$

Thus, the normalized eigenfunctions are

$$
\psi_{1}(x)= \pm \frac{1}{2}(1+\sqrt{3} x) \text { and } \psi_{2}(x)= \pm \frac{1}{2}(1-\sqrt{3} x)
$$

corresponding to eigenvalues $\lambda_{1}=\frac{\sqrt{3}}{2}$ and $\lambda_{2}=-\frac{\sqrt{3}}{2}$. Notice that the sign (or phase) of the eigenfunctions remains arbitrary.

Explicit solutions are not so readily obtained when the kernel is not separable. Therefore, one approach to dealing with a non-separable kernel is to approximate it with one that is separable. An obvious choice is to expand $K(x, y)$ in terms of an appropriate complete set $\left\{u_{j}(x)\right\}$,

$$
K(x, y)=\sum_{j=1}^{\infty} u_{j}(x) v_{j}(y),
$$

and then retain only the first $N$ terms.

\subsection{Solution by Iteration: Neumann Series}

A second approach to solving equations with non-separable kernels is to use a method of successive approximations. The method applies equally well to Fredholm and Volterra equations but, for the sake of specificity, we shall demonstrate it using the Fredholm equation

$$
\psi(x)=f(x)+\lambda \int_{a}^{b} K(x, y) \psi(y) d y .
$$

We start by approximating the unknown function $\psi(x)$ by

$$
\psi_{0}(x)=f(x)
$$

which, when substituted under the integral in (13.5.1), gives us the improved approximation

$$
\psi_{1}(x)=f(x)+\lambda \int_{a}^{b} K(x, y) f(y) d y
$$


A still better approximation $\psi_{2}(x)$ is obtained by replacing the $\psi(x)$ under the integral in (13.5.1) by $\psi_{1}(x)$ :

$$
\psi_{2}(x)=f(x)+\lambda \int_{a}^{b} K\left(x, y_{1}\right) f\left(y_{1}\right) d y_{1}+\lambda^{2} \int_{a}^{b} \int_{a}^{b} K\left(x, y_{1}\right) K\left(y_{1}, y_{2}\right) d y_{1} d y_{2} .
$$

Repeating this process of substituting the new $\psi_{n}(x)$ under the integral in (13.5.1), we develop the sequence

$$
\psi_{m}(x)=f(x)+\sum_{j=1}^{m} \lambda^{j} u_{j}(x)
$$

where

$$
u_{j}(x)=\int_{a}^{b} \int_{a}^{b} \cdots \int_{a}^{b} K\left(x, y_{1}\right) K\left(y_{1}, y_{2}\right) \cdots K\left(y_{j-1}, y_{j}\right) f\left(y_{j}\right) d y_{j} \cdots d y_{1} .
$$

Thus, the solution to our integral equation should be the limit

$$
\psi(x)=\lim _{m \rightarrow \infty} \psi_{m}(x)=f(x)+\lim _{m \rightarrow \infty} \sum_{j=1}^{\infty} \lambda^{j} u_{j}(x)=f(x)+\sum_{j=1}^{\infty} \lambda^{j} u_{j}(x)
$$

provided that the infinite series converges. This is called the Neumann series for $\psi(x)$ and its convergence occurs only for sufficiently small $\lambda, \lambda<\lambda_{\min }$ where $\lambda_{\min }$ is the magnitude of the eigenvalue of smallest magnitude of the corresponding homogeneous equation (i.e. when $f(x) \equiv 0$ ) .

As an application of the Neumann method of solution by iteration, let us consider the integral equation

$$
\psi(x)=x+\frac{1}{2} \int_{-1}^{1}(y-x) \psi(y) d y
$$

In this case, $\lambda=\frac{1}{2}$ and, as was noted in the example at the end of the preceding Section, $\lambda_{\min }=\frac{\sqrt{3}}{2}$. Therefore, we expect the Neumann series to converge. From (13.5.5), we find that the series is

$$
\begin{aligned}
& \psi_{0}(x)=x \\
& \psi_{1}(x)=x+\frac{1}{3} \\
& \psi_{2}(x)=x+\frac{1}{3}-\frac{x}{3} \\
& \psi_{3}(x)=x+\frac{1}{3}-\frac{x}{3}-\frac{1}{3^{2}}
\end{aligned}
$$


and, by induction,

$$
\psi_{2 m}(x)=x+\sum_{j=1}^{m}(-1)^{j-1} \frac{1}{3^{j}}-x \sum_{j=1}^{m}(-1)^{j-1} \frac{1}{3^{j}}
$$

with a similar expression for $\psi_{2 m+1}(x)$. The series in (13.5.7) does indeed converge in the limit as $m \rightarrow \infty$ and results in the solution

$$
\psi(x)=\frac{3}{4} x+\frac{1}{4}
$$

A more interesting application arises in connection with the scattering problem that we used to introduce this Chapter. The integral equation to be solved in that context is

$$
\psi(\boldsymbol{r})=e^{i \boldsymbol{k}_{0} \cdot \boldsymbol{r}}-\int_{\text {all }} \frac{e_{\text {space }}^{i k\left|\boldsymbol{r}-\boldsymbol{r}^{\prime}\right|}}{\left|\boldsymbol{r}-\boldsymbol{r}^{\prime}\right|} U\left(\boldsymbol{r}^{\prime}\right) \psi\left(\boldsymbol{r}^{\prime}\right) d V^{\prime} .
$$

The Neumann series solution of this equation is

$$
\begin{aligned}
\psi(\boldsymbol{r}) & =e^{i \boldsymbol{k}_{0} \cdot \boldsymbol{r}}+\int_{\text {all }} \frac{e^{i k\left|\boldsymbol{r}-\boldsymbol{r}^{\prime}\right|}}{\left|\boldsymbol{r}-\boldsymbol{r}^{\prime}\right|} U\left(\boldsymbol{r}^{\prime}\right) e^{i \boldsymbol{k}_{0} \cdot \boldsymbol{r}^{\prime}} d V^{\prime} \\
& +\int_{\text {all }} \int \frac{e^{i k\left|\boldsymbol{r}-\boldsymbol{r}^{\prime}\right|}}{\left|\boldsymbol{r}-\boldsymbol{r}^{\prime}\right|} U\left(\boldsymbol{r}^{\prime}\right) \frac{e^{i k\left|\boldsymbol{r}^{\prime}-\boldsymbol{r}^{\prime \prime}\right|}}{\left|\boldsymbol{r}^{\prime}-\boldsymbol{r}^{\prime \prime}\right|} U\left(\boldsymbol{r}^{\prime \prime}\right) e^{i \boldsymbol{k}_{0} \cdot \boldsymbol{r}^{\prime \prime}} d V^{\prime} d V^{\prime \prime}+\ldots
\end{aligned}
$$

In quantum mechanics, this series in powers of the potential $U(\boldsymbol{r})$ is called a perturbation series and its convergence depends on the strength of the interaction.

\subsection{Hilbert Schmidt Theory}

We shall now focus on the homogeneous Fredholm equation of the second kind

$$
\psi(x)=\lambda \int_{a}^{b} K(x, y) \psi(y) d y
$$

which, as we noted in Section 13.4, is also an eigenvalue equation. We shall assume that the kernel is Hermitian,

$$
K(x, y)=K^{\star}(y, x) .
$$

Our first objective is to prove that the eigenvalues, $\lambda_{m}$, are real and that the corresponding eigenfunctions, $\psi_{m}(x)$, are orthogonal. Let $\psi_{m}(x)$ and $\psi_{n}(x)$ be two different eigenfunctions and $\lambda_{m}$ and $\lambda_{n}$ be the corresponding eigenvalues. Then, according to (13.6.1), we have

$$
\psi_{m}(x)=\lambda_{m} \int_{a}^{b} K(x, y) \psi_{m}(y) d y, \text { and }
$$




$$
\psi_{n}(x)=\lambda_{n} \int_{a}^{b} K(x, y) \psi_{n}(y) d y
$$

If we complex conjugate the first of these and multiply it by $\lambda_{n} \psi_{n}(x)$ while multiplying the second by $\lambda_{m}^{\star} \psi_{m}^{\star}(x)$ and then integrate with respect to $x$, the two equations become

$$
\begin{gathered}
\lambda_{n} \int_{a}^{b} \psi_{m}^{\star}(x) \psi_{n}(x) d x=\lambda_{m}^{\star} \lambda_{n} \int_{a}^{b} \int_{a}^{b} K^{\star}(x, y) \psi_{m}^{\star}(y) \psi_{n}(x) d y d x, \text { and } \\
\lambda_{m}^{\star} \int_{a}^{b} \psi_{m}^{\star}(x) \psi_{n}(x) d x=\lambda_{m}^{\star} \lambda_{n} \int_{a}^{b} \int_{a}^{b} K(x, y) \psi_{n}(y) \psi_{m}^{\star}(x) d y d x .
\end{gathered}
$$

Invoking the symmetry property (13.6.2), we can rewrite (13.6.5) as

$$
\lambda_{m}^{\star} \int_{a}^{b} \psi_{m}^{\star}(x) \psi_{n}(x) d x=\lambda_{m}^{\star} \lambda_{n} \int_{a}^{b} \int_{a}^{b} K^{\star}(y, x) \psi_{m}^{\star}(x) \psi_{n}(y) d y d x .
$$

Subtracting (13.6.6) from (13.6.4), we have

$$
\left(\lambda_{n}-\lambda_{m}^{\star}\right) \int_{a}^{b} \psi_{m}^{\star}(x) \psi_{n}(x) d x=0 .
$$

When $m=n$, this becomes

$$
\left(\lambda_{m}-\lambda_{m}^{\star}\right) \int_{a}^{b}\left|\psi_{m}(x)\right|^{2} d x=0 .
$$

The integral cannot vanish and so we conclude that

$$
\lambda_{m}^{\star}=\lambda_{m}
$$

the eigenvalues are real. Notice that if the kernel were anti-Hermitian, $K(x, y)=$ $-K^{\star}(x, y)$, or even real skew-symmetric, we would have found $\lambda_{m}^{\star}=-\lambda_{m}$ which implies eigenvalues that are pure imaginary.

For $\lambda_{m} \neq \lambda_{n}$ when $m \neq n$, the integral has to vanish which means that the eigenfunctions are mutually orthogonal:

$$
\int_{a}^{b} \psi_{m}^{\star}(x) \psi_{n}(x) d x=0 \text { for } m \neq n .
$$


For $\lambda_{m}=\lambda_{n}$ when $m \neq n$, the eigenvalue is degenerate. Equation (13.6.7) is then indeterminate but the eigenfunctions can be orthogonalized by the Schmidt orthogonalization method.

Thus the outcome of a Hilbert-Schmidt eigenvalue problem involving an integral equation with a Hermitian kernel is just like that of a Sturm-Liouville problem with a self-adjoint differential operator. This similarity is of course no accident. The solution of the latter can always be expressed as

$$
\psi(x)=\lambda \int_{a}^{b} G(x ; y) \rho(y) \psi(y) d y
$$

where $G(x ; y)$ is the Green's function associated with $\mathfrak{L} \equiv \frac{d}{d x}\left(p(x) \frac{d}{d x}\right)+q(x)$ together with appropriate homogeneous boundary conditions at $x=a$ and $b$ and $\rho(x)$ is the weight function in the Sturm-Liouville equation

$$
\mathfrak{L} \psi(x)=-\lambda \rho(x) \psi(x) .
$$

As we know, this Green's function satisfies the requirement $G(x ; y)=G^{\star}(y ; x)$ and so we obtain a properly symmetrized Hilbert-Schmidt equation by introducing the function $\varphi(x)=\sqrt{\rho(x)} \psi(x)$ since it converts (13.6.10) to read

$$
\varphi(x)=\lambda \int_{a}^{b} G(x ; y) \sqrt{\rho(x) \rho(y)} \varphi(y) d y .
$$

The kernel is $K(x, y)=\sqrt{\rho(x) \rho(y)} G(x ; y)$.

The eigenfunctions of a Hilbert-Schmidt integral equation form a complete set in the sense that any function $g(x)$ that can be expressed by the integral

$$
g(x)=\int_{a}^{b} K(x, y) h(y) d y
$$

in which $h(y)$ is a piecewise continuous function, can be represented by the eigenfunction expansion

$$
g(x)=\sum_{m=1}^{\infty} c_{m} \psi_{m}(x) .
$$

Suppose that we extend this result to apply to the kernel itself and set

$$
K(x, y)=\sum_{m=1}^{\infty} c_{m}(x) \psi_{m}(y) .
$$

The coefficients $c_{m}(x)$ are

$$
c_{m}(x)=\int_{a}^{b} K(x, y) \psi_{m}^{*}(y) d y=\frac{1}{\lambda_{m}} \psi_{m}^{\star}(x)
$$


which yields the bilinear formula

$$
K(x, y)=\sum_{m=1}^{\infty} \frac{\psi_{m}^{\star}(x) \psi_{m}(y)}{\lambda_{m}} .
$$

We shall use this completeness to solve the non-homogeneous Fredholm equation

$$
\psi(x)=f(x)+\lambda \int_{a}^{b} K(x, y) \psi(y) d y
$$

The difference $\psi(x)-f(x)$ has an integral representation of the form (13.6.12) and so we can write

$$
\psi(x)-f(x)=\sum_{m=1}^{\infty} c_{m} \psi_{m}(x)
$$

where

$$
\begin{aligned}
c_{m} & =\int_{a}^{b} \psi_{m}^{\star}(x)(\psi(x)-f(x)) d x \\
& =\lambda \int_{a}^{b} \int_{a}^{b} \psi_{m}^{\star}(x) K(x, y) \psi(y) d y d x \\
& =\lambda \int_{a}^{b} \int_{a}^{b} \psi_{m}^{\star}(x) K(x, y) d x \psi(y) d y \\
& =\frac{\lambda}{\lambda_{m}} \int_{a}^{b} \psi_{m}^{\star}(y) \psi(y) d y .
\end{aligned}
$$

Adding and subtracting $f(y)$ to $\psi(y)$ under the integral, this becomes

$$
c_{m}=\frac{\lambda}{\lambda_{m}} \int_{a}^{b} \psi_{m}^{\star}(y)\{[\psi(y)-f(y)]+f(y)\} d y=\frac{\lambda}{\lambda_{m}} c_{m}+\frac{\lambda}{\lambda_{m}} \int_{a}^{b} \psi_{m}^{\star}(y) f(y) d y .
$$

Thus,

$$
c_{m}=\frac{\lambda}{\lambda_{m}-\lambda} \int_{a}^{b} \psi_{m}^{\star}(y) f(y) d y
$$

and the solution of the integral equation (13.6.14) is

$$
\psi(x)=f(x)+\sum_{m=1}^{\infty} \psi_{m}(x) \frac{\lambda}{\lambda_{m}-\lambda} \int_{a}^{b} \psi_{m}^{\star}(y) f(y) d y .
$$


This does not come as a surprise given our observation above regarding the similarity of the Hilbert-Schmidt and Sturm-Liouville theories. Note once again that if $\lambda=\lambda_{m}$ for some $m$ the non-homogeneous problem has no solution unless $f(x)$ is orthogonal to all of the (degenerate) eigenfunctions $\psi_{m}(x)$ that correspond to $\lambda_{m}$.

Example: To illustrate the use of Hilbert-Schmidt theory, we shall consider the nonhomogeneous Fredholm equation

$$
\psi(x)=f(x)+\lambda \int_{-1}^{1}(y+x) \psi(y) d y
$$

The eigenvalues and eigenfunctions for this kernel and interval of integration were found in Section 13.4. The normalized eigenfunctions are

$$
\psi_{1}(x)= \pm \frac{1}{2}(1+\sqrt{3} x) \text { and } \psi_{2}(x)= \pm \frac{1}{2}(1-\sqrt{3} x)
$$

corresponding to eigenvalues $\lambda_{1}=\frac{\sqrt{3}}{2}$ and $\lambda_{2}=-\frac{\sqrt{3}}{2}$. Thus, substituting into (13.6.15) we have

$$
\begin{aligned}
\psi(x)= & f(x)+\sum_{m=1}^{2} \psi_{m}(x) \frac{\lambda}{\lambda_{m}-\lambda} \int_{-1}^{1} \psi_{m}(y) f(y) d y \\
= & f(x)+\frac{1}{4} \frac{1+\sqrt{3} x}{\frac{\sqrt{3}}{2}-\lambda} \lambda \int_{-1}^{1}(1+\sqrt{3} y) f(y) d y \\
& +\frac{1}{4} \frac{1-\sqrt{3} x}{-\frac{\sqrt{3}}{2}-\lambda} \lambda \int_{-1}^{1}(1-\sqrt{3} y) f(y) d y .
\end{aligned}
$$

At this point, we need a specific functional form for $f(x)$. We choose $f(x)=x^{2}$.

Clearly, this is not orthogonal to either eigenfunction and so there will be no solution for $\lambda=\lambda_{1}$ or $\lambda=\lambda_{2}$. Substituting into the two integrals, we find

$$
\int_{-1}^{1}(1 \pm \sqrt{3} y) y^{2} d y=\frac{2}{3}
$$

and so

$$
\psi(x)=x^{2}+\frac{2 \lambda}{3} \frac{3 x+2 \lambda}{3-4 \lambda^{2}}, \quad \lambda \neq \pm \frac{\sqrt{3}}{2} .
$$

Explicit substitution into the original Fredholm equation comfirms that this is indeed the solution. For the specific value of $\lambda=\frac{1}{2}$ it becomes

$$
\psi(x)=x^{2}+\frac{x}{2}+\frac{1}{6} .
$$


Notice that if we add $\pm \frac{x}{\sqrt{3}}$ to $x^{2}$ we obtain an $f(x)$ that is orthogonal to one of the eigenfunctions. In particular, $f(x)=x^{2}-\frac{x}{\sqrt{3}}$ is orthogonal to $\psi_{1}(x)$ while $x^{2}+\frac{x}{\sqrt{3}}$ is orthogonal to $\psi_{2}(x)$. Choosing the former, we find

$$
\int_{-1}^{1}(1-\sqrt{3} y)\left(y^{2}-\frac{y}{\sqrt{3}}\right) d y=\frac{4}{3}
$$

and so

$$
\psi(x)=x^{2}-\frac{x}{\sqrt{3}}-\frac{2 \lambda}{3} \frac{1-\sqrt{3} x}{\sqrt{3}+2 \lambda}, \quad \lambda \neq-\frac{\sqrt{3}}{2} .
$$

In particular, if $\lambda=\lambda_{1}=\frac{\sqrt{3}}{2}$,

$$
\psi(x)=f(x) \mp \frac{1}{3} \psi_{2}(x)=x^{2}-\frac{\sqrt{3} x}{6}-\frac{1}{6} .
$$

Again, explicit substitution into the non-homogeneous Fredholm equation confirms that this is the solution. 